Naturwissenschaften 58, 312-318 (1971)

(C) by Springer-Verlag 1971 


\title{
Warmwasser-Fischzucht Neue Verfahren der Aquakultur
}

\author{
Ch. Meske \\ Max-Planck-Institut für Kulturpflanzenzüchtung, Ahrensburg *
}

\begin{abstract}
New methods for keeping, feeding and breeding carp and other fish in running warm water are described. In a closed circuit ("system Ahrensburg") the water is recycled through a biological clarifying plant and is maintained at a constant temperature, thus enabling the employment of these new methods independently of environmental pollution. The development of weight and of sexual maturity is very fast, which makes experiments on the biology of reproduction and breeding much easier. Warm-water pisciculture opens up new possibilities for the production of marketable fish.
\end{abstract}

Seit Jahrtausenden werden wildlebende Fische mit Netzen, Reusen, Angeln usw. aus den Meeren, Flüssen und Seen gefischt. Erst in jüngster Zeit beginnt sich die Erkenntnis durchzusetzen, daß dieser „Fischerei" natürliche Grenzen gesetzt sind. Schon zeigen sich erste Anzeichen der „Überfischung im Meer. Die Lachsfänge in der Ostsee sind von 1964/65 bis 1968/69 um ca. $50 \%$ zurückgegangen [1]. Im Nordatlantik nahmen die Heringsfänge von 1967 bis 1969 um ca. $45 \%$ ab [2], und zum ersten Male ist bereits der Weltfischereiertrag rückläufig [3]. In den Binnengewässern haben zunehmende Gewässerverschmutzung zu katastrophalen Fischsterben geführt. Mit Ausnahme der Teichwirtschaften verharrt die herkömmliche Fischerei aber noch im Stadium des Sammlers und Jägers.

Auf dem Lande sind diese Urformen des menschlichen Nahrungserwerbs seit frühester Zeit durch die Agrikultur abgelöst worden. Analog hierzu beginnt man seit wenigen Jahren, die Aquakultur als Sammelbegriff für Methoden zur Erzeugung von nützlichen Wasserorganismen $\mathrm{zu}$ verwenden. Man erkennt allmählich, daß nicht nur ständig geerntet werden kann, sondern daß z.B. auch Fische in Kultur genommen und produziert werden müssen, wenn man dem steigenden Eiweißbedarf der Weltbevölkerung einerseits und den Gefahren des Úberfischens und der Umweltverschmutzung andererseits begegnen will. Solche Aquakulturen, in denen Fische, aber auch andere Wassertiere, z.B. Muscheln oder Krebse, vermehrt, gefüttert und schließlich dem Verbrauch zugeführt werden, werden bei Anhalten der bisher beobachteten Tendenzen die Fischerei herkömmlicher Art ablösen.

\section{Begritf}

Die Warmwasser-Fischzucht ist der modernste Zweig der Aquakultur. Die grundlegenden Arbeiten hierzu wurden 1964-1970 im Max-Planck-Institut für Kulturpflanzenzüchtung in Ahrensburg bei Hamburg ausgeführt. Den AnstoB gab die Idee von Sengbuschs, Karpfen ohne Zwischenmuskelgräten zu züchten [4]; eine Aufgabe, die von Sengbuschs früheren Arbeiten

\footnotetext{
* Seit 1.1.1971 BFA für Fischerei, Außenstelle Ahrensburg.
}

auf dem Gebiet der Kulturpflanzenzüchtung - alkaloidfreie Lupine, anthozyanfreier Spargel, lamellenloser Champignon u.a. - gedanklich verwandt war [5]. Die geplante züchterische Arbeit an Karpfen setzte jedoch eine kontrollierbare Haltung, Aufzucht, Fütterung und Vermehrung voraus. In Freilandgewässern sind die Voraussetzungen hierfür nicht gegeben. Stark schwankende, meist viel zu niedrige Wassertemperaturen, Krankheitserreger, Fischräuber und Wasserverunreinigung erlauben dort weder Arbeiten der angewandten Genetik noch physiologische Experimente. Ein „Raumfaktor", über den in der fischereiwissenschaftlichen Literatur seit Jahrzehnten geschrieben wird, verhinderte bislang alle Aquarienversuche an Nutzfischen, da er angeblich eine wachstumshemmende Wirkung in kleinen Behältern ausüben würde $[6,7]$. Wir bewiesen, da $\beta$ es möglich ist, Karpfen und andere Fischarten auf engstem Raum aufzuziehen und durch temperiertes Wasser hierbei Zuwachsraten zu erreichen, die weit über mitteleuropäischen Freilandwerten liegen [8-10]. Die entscheidenden Voraussetzungen für die schnelle Gewichtsentwicklung der Fische sind:

1. Ständiger Wasseraustausch in den Aquarien - dadurch Aufheben jeder "Raumfaktor-Wirkung" und Zuwachs unabhängig von der Beckengröße.

2. Ganzjährige Warmwasserhaltung - welche ganzjährigen Zuwachs und früheren Eintritt der Laichreife ergibt.

3. Ausreichende Ernährung, möglichst ausschließlich mit Trockenfuttermitteln - hierdurch wird die ganzjährige Haltung und Aufzucht ermöglicht und die kontinuierliche Forschungsarbeit gewährleistet.

Als einen der entscheidenden Faktoren der Warmwasser-Fischzucht erwähnte ich den ständigen Wasseraustausch in den Fischbehältern. Die Menge des Wasserdurchstromes steht in direkter Proportion zur Masse der im Becken gehaltenen Fische. Qualitativ geeignetes Wasser vorausgesetzt, kann nach eigenen Erfahrungen bei $23{ }^{\circ} \mathrm{C}$ Wassertemperatur ca. 1 Liter Wasser/min/kg Fisch als notwendige Durchflußmenge angenommen werden. Der hierbei anzustrebende Sauerstoffgehalt von ca. $7 \mathrm{mg} /$ Liter wird gegebenenfalls durch zusätzliche Belüftung der Aquarien erzielt. Es läßt sich im Fischbecken so ein Besatz von ca. 1:10, 
d.h. ein Kilogramm Fisch auf 10 Raumliter, als Durchschnittswert angeben. Engerer Besatz bis auf $1: 3$ ist möglich. Für Brut gelten andere Werte [11]. Für Experimente in kleinerem Rahmen kann das benötigte Wasser dem Leitungsnetz entnommen werden und nach Aufheizung durch die Versuchsaquarien geführt und anschließend abgeleitet werden. Die großen Wassermengen, die für eine Intensivhaltung unter Praxisbedingungen benötigt werden, fallen in Europa nur im AnschluB an größere Industriebetriebe, bes. Kraftwerke, billig und temperiert an. In den Tropen stehen hingegen ungeheure Mengen warmen Wassers ständig zur Verfügung, z.B. am Amazonas. Die europäischen Großindustrien, ganz besonders die Kernkraftwerke, die sehr große Kühlwassermengen benötigen, entnehmen dieses fast immer Flüssen. Mit der zunehmenden Verschmutzung unserer Gewässer vermindert sich die Möglichkeit, solches Kühlwasser für Zwecke der Warmwasser-Fischzucht zu verwenden. Würde man große Fischmastanstalten im Anschluß an Kernkraftwerke bauen - was technisch durchaus möglich ist so bestünde immer das Risiko eines plötzlichen Totalverlustes durch akute Fischvergiftungen infolge Gewässerverschmutzung. Das Fischsterben im Rhein vor zwei Jahren ist ein warnendes Beispiel.

\section{Umlaufverfahren}

Wir konnten in den letzten Jahren ein Verfahren zur Haltung und Aufzucht von Karpfen und anderen Nutzfischen entwickeln, das die Risiken der Gewässerverunreinigung ausschließt. Es handelt sich hierbei um ein geschlossen arbeitendes System, in dem die Fische ständig das gleiche Wasser angeboten bekommen, das nach Passieren der Aquarien biologisch aufbereitet worden ist. Fig. 1 gibt ein Schema dieses

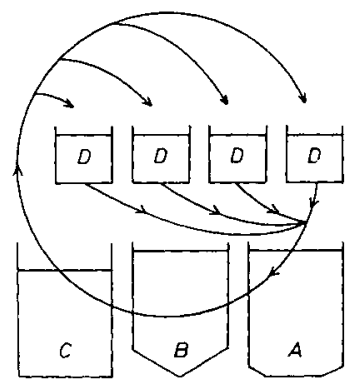

Fig. 1. Schema des Wasserkreislaufes (Erklärung im Text)

Wasserkreislaufes wieder. Das aus den Aquarien $D$ abfließende verschmutzte Wasser wird in einem Belebtschlammbecken $A$ einer biologischen Reinigung unterzogen, durchfließt ein Nachklärbecken $B$ und wird aus dem Pumpbecken $C$ wieder zu den Fischbecken geleitet. Die Entwicklung dieses Systems benötigte mehrere Jahre, bis wir solche Kreisläufe als Vorläufer halbtechnischer Anlagen ansehen konnten. Das Wasser soll weitestgehend frei von schädlichen Eiweißabbauprodukten und möglichst klar in die Aquarien gelangen. Beides ist uns bisher mit Hilfe von zahlreichen technischen Details soweit gelungen, daß im Hinblick auf die wissenschaftliche und praktische Bedeutung des Verfahrens eine nähere Beschreibung unerläßlich ist. Fig. 2 zeigt in Aufsicht und Querschnitt die Wasser- aufbereitungstechnik einer unserer derzeit arbeitenden Umlaufanlagen, die die institutsinterne Bezeichnung „Kreislauf 601" trägt.

Durch ein Rohr 1 fließt das aus den Fischbecken abgeleitete Wasser in das Belebtschlammbecken $A$. Eine zentrale Luftleitung 2 , die von einem - nicht gezeichneten - Luftverdichter gespeist wird, führt über das Rohr 3 Frischluft in den Rohrbelüfter 4. Aus diesem wird die Luft feinperlig in den Belebtschlamm geblasen. Sie dient zur Sauerstoffversorgung der aeroben Mikroorganismen und zur Erzeugung einer rotierenden Umwälzung des Wasser-Schlamm-Gemisches, wobei die Abweiswände 5 richtungsgebend sind. Hinter der Tauchwand 6 steigt der verdünnte Schlamm an und läuft über die Überlaufwand 7 hinter der Tauchwand 8 in das Nachklärbecken $B$. Hier trennt sich das im Becken $A$ weitgehend aufbereitete Wasser vom Schlamm, der nach unten sinkt. Dieser absinkende Schlamm wirkt auch als Filter und hält feine Partikel aus dem Wasser zurück. Aus dem Pumpensumpf 9 wird der Schlamm ständig durch die mit Luft betriebene Mammutpumpe 10 durch den Schlammabschneider 11 in das Becken $A$ zurückgeführt. Im siebkastenförmigen Schlammabscheider kann ein Teil des Überschußschlammes verlanden und dann entfernt werden. (Reicht die Verlandung nicht aus und wächst der Schlamm in Becken $B$ zu hoch an, wird dieser mit einem nicht eingezeichneten Filter entnommen und verworfen.) Das Oberflächenwasser flieBt aus dem Nachklärbecken über die Überlaufrinne 12 in die Zisterne $C$. Hier führt das Rohr 13 Frischluft über den Rohrbelüfter 14 in das geklärte Wasser, und der Plattenheizkörper 15 sorgt in Verbindung mit dem Thermostaten 16 für die Einhaltung der gewählten Wassertemperatur. Die Wasserpumpe 17 fördert das Reinwasser im Rohr 18 über die Druckleitung $19 \mathrm{zu}$ den Fischbehältern. Hiermit ist der Wasserkreislauf geschlossen.

Die eingezeichneten Teile 20 bis 25 dienen der mehrmals am Tage nötigen Wartung der Kläranlage, wobei es darauf ankommt, den sich an den Wänden des Beckens $B$ ansetzenden Schlamm abzulösen und zur erneuten Verarbeitung dem Belebtschlammbecken $A$ zuzuführen. Hierzu werden die Ventile 20 so umgeschaltet, daß durch das Rohr 21 soviel Oberflächenwasser aus dem Becken $B$ durch das Druckrohr 22 in Becken $C$ gepumpt wird, bis der Wasserspiegel im Becken $B$ unter die Mündung des Rohres 21 gesenkt ist. Jetzt wird die Pumpe 17 abgeschaltet, das Ventil 25 geöffnet und durch starke Luftzufuhr durch das Rohr 23 die an den Wandungen des Beckens $B$ anhaftenden Schlammpartikel abgelöst und aufgewirbelt. Die Mammutpumpe 10 pumpt nun auch diesen Schlamm zur weiteren Aufbereitung in Becken $A$ zurück. Nach einigen Minuten wird das Ventil 25 wieder geschlossen, der Schlamm in Becken $B$ trennt sich durch Schwerkraft vom Wasser, und nach weiteren 5 min kann durch Umschalten der Ventile 20 und Einschalten der Pumpe 17 der Kreislauf wieder angefahren werden. Nach dem hier wiedergegebenen Prinzip arbeiten bei uns mehrere Kreisläufe. Sie unterscheiden sich lediglich in der GröBe oder auch in der Form der Becken. Zur Zeit bauen wir eine Neuanlage von insgesamt ca. $350 \mathrm{~m}^{3}$ Klärbeckenvolumen, die in mehrere Kreisläufe getrennt werden. Die grundlegenden konstruktiven Merkmale unserer Wasseraufbereitung orientie- 


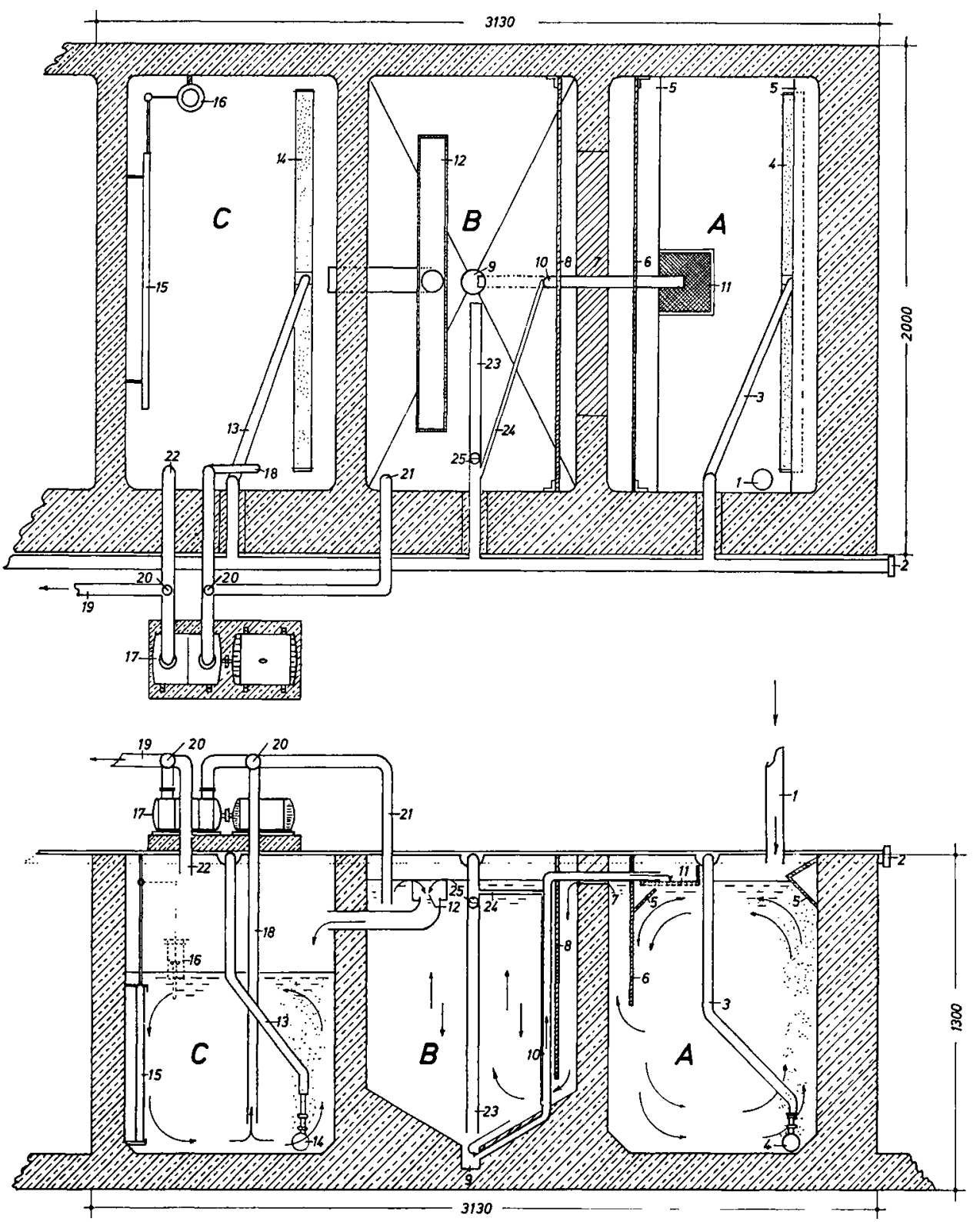

Fig. 2. Aufsicht (oben) und Schnitt (unten) der Wasseraufbereitungsanlage im Kreislauf 601 (Erklärung im Text). Maßangaben in $\mathrm{mm}$

ren sich an den von der Industrie zur Abwasseraufbereitung hergestellten Anlagen. Die Abbauleistung solcher sog. vollbiologischer Kläreinheiten, wie sie z.B. zur Reinigung kommunaler Abwässer verwendet werden, sind jedoch mit unserem System nicht zu vergleichen:

1. Bei herkömmlichen Kläranlagen passiert das zu reinigende Abwasser alle Teile der Anlage nur einmal. Im Ablauf wird kein total aufbereitetes Wasser erwartet. Demgegenüber wird bei unserem Kreislaufsystem das zirkulierende Wasser immer wieder gereinigt.

2. Die städtischen und industriellen Kläranlagen benötigen eine in Einwohnergleichwerten gemessene Mindestbelastung des anfallenden Abwassers, um die Mikroorganismen des Belebtschlammes genügend mit Nahrung zu versorgen. Das aus den Aquarien unserer Anlagen abfließende Wasser ist im Vergleich hierzu nährstoffarm. Eine im herkömmlichen Durchflußprinzip arbeitende Kläranlage würde ausschließlich mit
Aquarienwasser nicht arbeiten können, da der Belebtschlamm ,verhungern" würde. Dies gilt besonders in Zeiten geringer Belastung, z.B. während der nächtlichen Fütterungspause. Hingegen werden im kontinuierlich betriebenen Umlaufverfahren die nach einmaligem Passieren der biologischen Klärung nicht aufbereiteten Stoffe den Mikroorganismen ständig wieder zugeführt.

3. Für die herkömmlichen, im Durchlauf arbeitenden Kläranlagen sind Mindestzeiten vorgeschrieben, die das verunreinigte Wasser in den einzelnen Zonen der Anlage, besonders im Belebtschlammbecken, zubringen muB. Erst nach einer solchen Mindestverweilzeit kann der geforderte Reinigungsgrad garantiert werden. Hierdurch ist in Abhängigkeit von den normalerweise anfallenden Wassermengen die Größe der Becken solcher Anlagen bedingt. Das von uns entwickelte Prinzip der geschlossenen Wasserführung im Kreislauf mit biologischer Klärung kann infolge der ständig wiederholten Passagen mit erheblich geringeren Bek- 
kenvolumina auskommen. Bei jedem Wasserdurchsatz wird hierbei nur eine Teilklärung erwartet und benötigt; durch die Wiederholung der Aufbereitungsvorgänge übertrifft aber die Kreislaufkonstruktion die Durchlaufanlagen an Abbauintensität bei weitem.

4. Alle Anlagen mit biologischer Wasseraufbereitung arbeiten in Abhängigkeit von der Außentemperatur bzw. von der davon meist abhängigen Abwassertemperatur. Unser Kreislauf enthält als wesentliches Merkmal der Warmwasser-Fischzucht eine Heizung, die in Verbindung mit einem Thermostaten die Einhaltung der gewählten Wassertemperatur vornimmt. Bei der von uns im Hinblick auf eine gute Gewichtsentwicklung bevorzugten ganzjährigen Temperatur von $23{ }^{\circ} \mathrm{C}$ liegen offenbar Verhältnisse im Belebtschlamm vor, die sich von denen in unbeheizten Freilandkläranlagen wesentlich unterscheiden. Dies gilt sowohl für das Spektrum der Mikroorganismen als auch für deren Abbauleistungen.

Da bei unserem Verfahren erstmalig eine biologische Klärung im Kreislauf eingebaut ist und diese erstmalig beheizt wird, sind zahlreiche Untersuchungen über die Zusammensetzung der Organismen des Belebtschlammes und deren Arbeit bei dieser Art der Wasseraufbereitung notwendig. Die eiweißhaltigen Schmutzstoffe, die bei der Fischhaltung das Wasser belasten, werden in dem oben beschriebenen Verfahren weitgehend abgebaut. Ein Beispiel hierfür gibt Fig. 3:

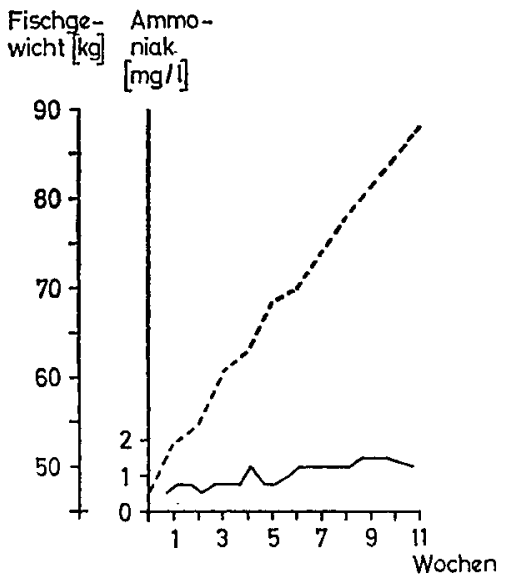

Fig. 3. Gewichtszunahme der Karpfen und Ammoniakwerte im Kreislauf 601. - - Fischgewicht, —— Ammoniak

Innerhalb eines Versuchszeitraumes von 11 Wochen stieg bei konstant zunehmendem Fischgewicht von $47,2 \mathrm{~kg}$ auf $90,0 \mathrm{~kg}$ der Ammoniakgehalt nicht über $1,5 \mathrm{mg} /$ Liter. Die nächsten Abbaustufen Nitrit und Nitrat erreichten im gleichen Zeitraum Maximalwerte von $2,4 \mathrm{mg} /$ Liter bzw. $120 \mathrm{mg} /$ Liter, der $\mathrm{O}_{2}$-Gehalt betrug im Mittel 7,25 mg/Liter. Der Frischwasserzusatz zum Kreislauf beträgt z.Z. noch ca. $1 \%$ seines Gesamtvolumes pro Tag als Ausgleich für die Verdunstung oder geschieht automatisch bei technischen Defekten der Pumpen. Wir haben in allen bisherigen Versuchen beobachtet, daß eine Aufsalzung des Wassers mit Nitrat als Folge der ständigen Eiweißzufuhr (Futter, Kot) toxische Grenzen nicht überschreitet. Offenbar laufen neben der Nitrifikation auch entgegengesetzte Prozesse innerhalb des Umlaufsystems ab. Wahrscheinlich findet die Denitrifikation, bei der
Stickstoff gasförmig entweicht, unter der Mitwirkung anaerober Mikroorganismen im Nachklärbecken statt. Die Kreislaufsysteme können mit einem durchschnittlichen Fischbesatz von 1:100 besetzt sein. Wir sprechen von einem Fisch:Wasser-Verhältnis von 1:100, bezogen auf das gesamte Umlaufwasser. Wir haben jedoch schon Werte von 1:50 erreicht, und begonnene Versuche mit veränderter Wasserführung deuten auf die Möglichkeit einer weiteren Verbesserung der Fisch: Wasser-Relation hin.

Für die Warmwasser-Fischzucht ist die Haltung der Fische in warmem, fließendem Wasser eines der eingangs genannten Kriterien. Die Haltung im Kreislaufsystem ist also keine conditio sine qua non, bietet aber sowohl wissenschaftlichen Untersuchungen am Fisch als auch in der Praxis sehr große Vorteile. Schon aus Fig. 1 ist ersichtlich, daß bei Parallelschaltung vieler Aquarien die Garantie für die Speisung aller Fischbehälter mit dem gleichen Wasser gegeben ist. Die für die Fische wichtigsten Umweltfaktoren, Temperatur und Wasserqualität, sind für alle Versuchstiere eines Kreislaufes gleich. Hierdurch bietet sich die Möglichkeit für zahlreiche Experimente, z. B. auf dem Gebiet der Ökologie, der Fortpflanzungsbiologie und der Ernährungsphysiologie. Die meisten Umweltfaktoren sind kontrollierbar und steuerbar. Für die Experimente mit warmblütigen Versuchstieren sind dies seit langem ebenso selbstverständliche Voraussetzungen wie bei der neuzeitlichen Intensivhaltung solcher Tiere (Schweine, Hühner). Für die experimentelle und praktische Arbeit mit Nutzfischen werden diese Möglichkeiten durch die Warmwasser-Umlaufanlage geschaffen.

\section{Fütterung und Zuwachs}

Die Ernährung der Nutzfische bei Warmwasserhaltung sollte ebenso kontrollierbar und steuerbar sein wie die Umweltfaktoren. Angestrebt werden müssen daher konfektionell gefertigte Alleinfuttermittel. Nach bisheriger Lehrmeinung benötigt der Karpfen einen Lebendfutteranteil von $50 \%$ seiner Gesamtnahrung [12]. Nur auf diese Weise könnte überhaupt Beifutter verwertet werden, da die Nährtiere die für die Aufbereitung des Zufutters notwendigen Enzyme liefern sollten [13]. Wir konnten diese Ansichten durch zahlreiche Versuche widerlegen und beweisen, daß die ausschließliche, ganzjährige Trockenfutterernährung des Karpfens möglich ist. Hierfür eignet sich z.B. pelletiertes Forellenfertigfutter mit einem Rohproteingehalt von $30-40 \%$ [14]. Auch der im Freiland als Pflanzenfresser bezeichnete Grasfisch (Ctenopharyngodon idella) läBt sich mit gleichartigem Futter ernähren [15]. Bei der Fütterung von Aalen haben wir zwar auch gute Erfahrungen mit Trockenfuttermitteln gemacht (Fig. 4), doch scheint bisher eine Kombinationsfütterung mit Fischabfällen zweckmäßiger.

Die Gewichtsentwicklung ist unter den geschilderten Bedingungen der Warmwasser-Fischzucht sehr schnell. Wir haben im Aquarium bei $23^{\circ} \mathrm{C}$ ganzjähriger Wassertemperatur Karpfen vom $\mathrm{Ei}$ an aufgezogen, die nach Vollendung des ersten Lebensjahres ca. $2 \mathrm{~kg}$ wogen. Der Karpfen in mitteleuropäischen Teichwirtschaften benötigt zum Erreichen dieses Gewichtes ca. 3,5 Jahre. Seine Gewichtsentwicklung ist durch kurze Wachstumsperioden in den Sommermonaten und 


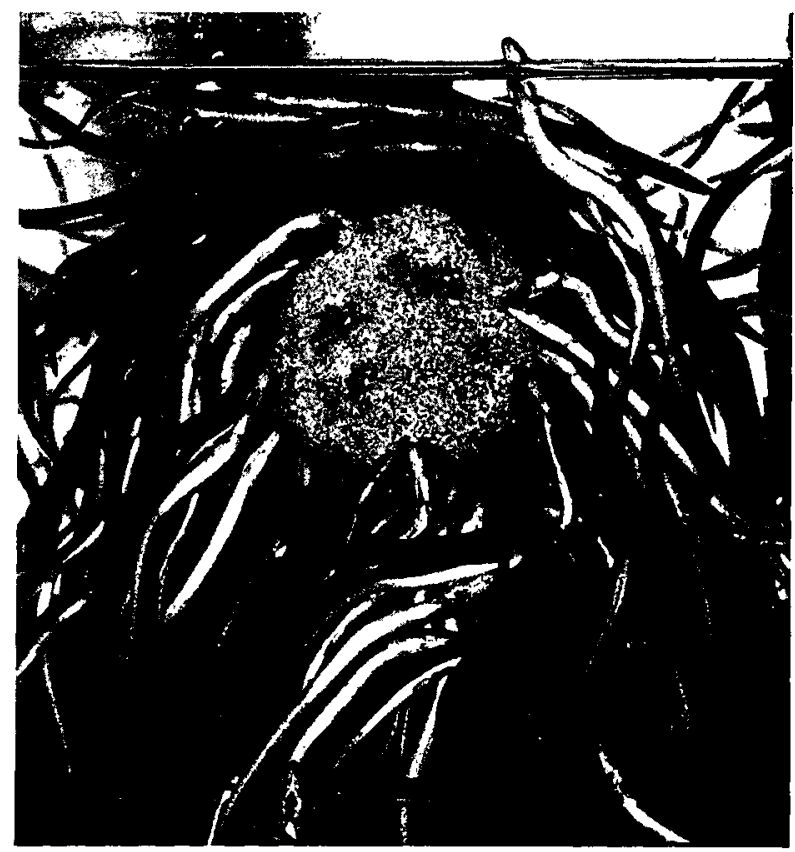

Fig. 4. Fütterung von Aalen im Aquarium mit Trockenfutter

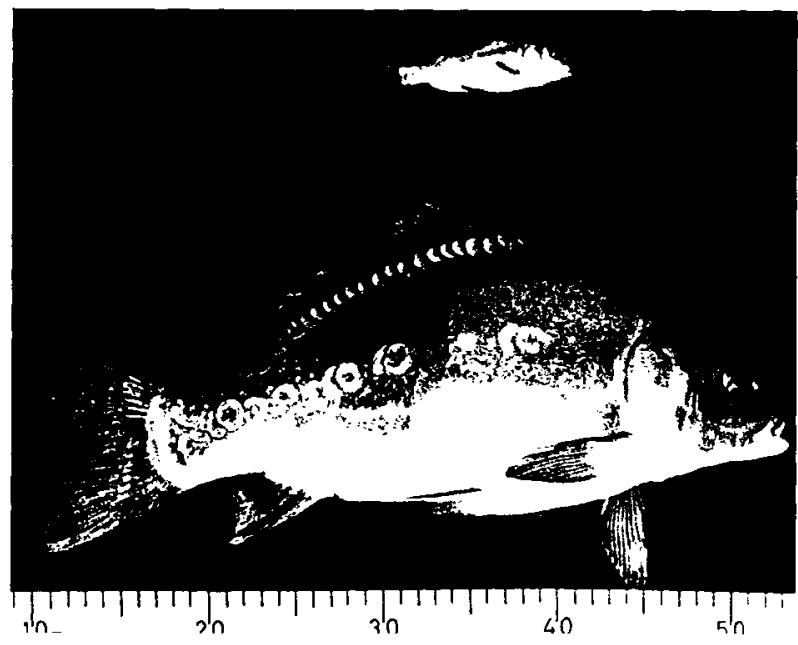

Fig. 5. Einjähriger Karpfen nach Teichhaltung (40 g, oben), einjähriger Karpfen nach Warmwasserhaltung (1750 g, unten)

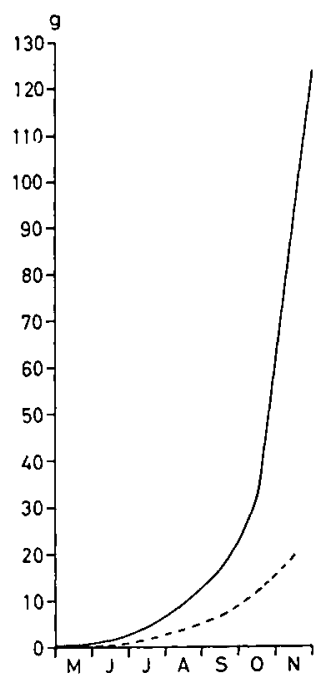

Fig. 6. Gewichtsentwicklung von Glasaalen Im Freiland und im Aquarium beobachtete Maximalzunahmen. - - Warmwasserhaltung, - - Freilandhaltung
Gewichtsstagnation oder -abnahme in den kühlen Jahreszeiten gekennzeichnet. Die Fig. 5 zeigt zwei Karpfen gleicher Zucht nach genau einem Lebensjahr bei Freilandháltung und bei Warmwasserhaltung. Das obere, ein im Teich gezogenes Exemplar, wiegt $40 \mathrm{~g}$, das untere, im Aquarium aufgezogene Tier $1750 \mathrm{~g}$. In den Aquarien unserer Ahrensburger Anlage leben Karpfen, die 2,5 Jahre alt $6 \mathrm{~kg}$ wiegen.

Ebenso zeigen Aale bei Warmwasserhaltung Gewichtszunahmen, die in dieser Höhe bisher nicht bekannt waren. Die an den westeuropäischen Küsten eintreffenden Glasaale haben nach dem derzeitigen Wissensstand eine ca. dreijährige Atlantikwanderung hinter sich und wiegen ca. $0,4 \mathrm{~g}$. Sie sollen nach einem Jahr Süßwasseraufenthalt erst $1 \mathrm{~g}$ wiegen [16]. Hingegen wurde in einem Teichversuch am Ende des ersten Sommers ein Maximalgewicht von $20 \mathrm{~g}$ beobachtet [17] und in unserem Warmwasseraquarium nach sieben Monaten ein Maximalgewicht von $124 \mathrm{~g}$ erreicht (Fig. 6) [18]. Vom Glasaalstadium an waren einige unserer Aale nach 22monatiger Warmwasserhaltung auf über $500 \mathrm{~g}$ Stückgewicht gewachsen. In der Literatur wird für ein solches Gewicht ein Alter von über 10 Jahren angegeben [16].

\section{Fortpflanzungsbiologie}

Bei ganzjähriger Warmwasserhaltung nehmen die Fische nicht nur ganzjährig zu, sie werden auch früher geschlechtsreif. Bei Karpfen konnten wir dies bei männlichen Tieren nach 3 Lebensmonaten, bei weiblichen nach 15 Lebensmonaten beobachten. In unseren Breitengraden werden Karpfen im Freiland im Durchschnitt nach 3-4 Jahren ( $\left.\hat{\sigma}^{\lambda}{ }^{\lambda}\right)$ bzw. nach 4-5 Jahren (우우) laichreif. Die Faktoren, die zur Vorverlegung der Geschlechtsreife führen, sind vor allem die konstante Wasserwärme von $23^{\circ} \mathrm{C}$ und die Verabreichung vollwertigen Futters. Die Faktoren, die im einzelnen zur Entwicklung der verschiedenen Reifestadien der Gonaden führen, sind noch weitgehend unaufgeklärt. In mitteleuropäischen Teichen laichen die Karpfen nach einem vorausgegangenen Laichspiel ab. Auslöser der im späten Frühling stattfindenden Balz sind wahrscheinlich steigende Wassertemparaturen und intensiveres Sonnenlicht bzw. zunehmende Tageslänge. Über die endokrinen Vorgänge, die Laichspiel und Laichabgabe auslösen, liegen bisher nur wenige Untersuchungen vor. Offenbar werden das letzte Eireifestadium und die Ovulationsauslösung durch die Ausschüttung gonadotroper Hormone der Hypophyse bewirkt [19].

Der Ablauf dieser Prozesse wird bei der Warmwasserhaltung durch die hier weitgehend konstanten Umweltverhältnisse beeinflußt. Wir wenden daher bei unseren Aquarienkarpfen eine Methode an, die diese hormonellen Vorgänge experimentell auslöst. Die Injektion von aufgeschwemmten Fischhypophysen führt hierbei zur Erreichung des letzten Reifestadiums und zur Ovulation. Die Hypophysierung wurde auf Grund vorbereitender Arbeiten anderer Autoren [20,21] von Woynarovich [22] speziell für die Karpfenvermehrung praxisreif gemacht. Sie wird hauptsächlich in den osteuropäischen Ländern in großem Stil zur planmäßigen Brutgewinnung bei Teichkarpfen angewendet. Wir haben diese Methode mit Erfolg bei in Warmwasser aufgezogenen Karpfen angewendet [23]. Die Hypo- 
physen, die intramuskulär injiziert werden, bewirken die Ovulation innerhalb von 24 Std. Dann können Eier und Milch durch Abstreifen gewonnen werden. Unter Zusatz bestimmter Substanzen, die die Quellung der Eier fördern und ihr Verkleben verhindern, werden die Geschlechtsprodukte, in einer Schale vermischt und die so befruchteten Eier in sog. Zugergläsern bei ständigem Wasserdurchfluß erbrütet. Das Schlüpfen der Karpfenlarven kann bei Wassertemperaturen über $22{ }^{\circ} \mathrm{C}$ schon nach $36-48$ Std. erfolgen. Die Brut können wir nahezu verlustfrei mit Larven von Artemia salina anfüttern und anschließend mit Trockenfutter großziehen [11].

Die Hypophysierung führt sowohl bei Teich- als auch bei Aquarienkarpfen nicht immer zur Laichgewinnung. Innerhalb eines bestimmten Versuchszeitraumes konnte die durchschnittliche Erfolgsquote von ca. $50 \%$ der bei Warmwasserhaltung hypophysierten Karpfen von uns jedoch durch vorhergehende Behandlung mit Gestagenen auf ca. 90\% erhöht werden [24]. Der von der Jahreszeit weitestgehend unabhängige Sexualzyklus bei Karpfen der Warmwasserhaltung [25] macht es möglich, zu allen Monaten des Jahres Brut zu erzeugen. Außerdem geben die Tiere mehrmals im Jahr Laich ab, und zwar jeweils mehrere hunderttausend Eier.

\section{Züchtung}

Die Methoden der Eigewinnung und Brutanzucht sind wichtige Voraussetzungen für züchterische Arbeiten. Der experimentelle Genetiker findet bei Fischen unter diesen Bedingungen zahlreiche Vorteile gegenüber den Arbeiten an Warmblütern. Die große Nachkommenszahl, die rasche Generationsfolge, die Unabhängigkeit von jahreszeitlichen Rhythmen und die Befruchtung in vitro sind als Komplex nur bei der WarmwasserFischzucht möglich. Fig. 7 zeigt einen Bastard von Karpfenweibchen und Goldfischmännchen als Beispiel für die Anwendbarkeit der Verfahren.

Die züchterische Bearbeitung der Karpfen ist teichwirtschaftlich kaum möglich. Für ein erfolgreiches Laichspiel werden einem Weibchen mehrere Männchen beigegeben. Dagegen erlaubt die Befruchtung in vitro gezielte Paarung von Einzelindividuen, die Erzeugung von gewünschten Nachkommen und die Bildung von Inzuchtlinien. Die kontrollierbare Aufzucht der Brut im Aquarium ermöglicht Nachkommenschaftsprüungen und bestimmte Leistungsvergleiche schon nach wenigen Wochen. Die generelle Standardisierung der Umweltbedingungen läßt für Nutzfische erstmals um-

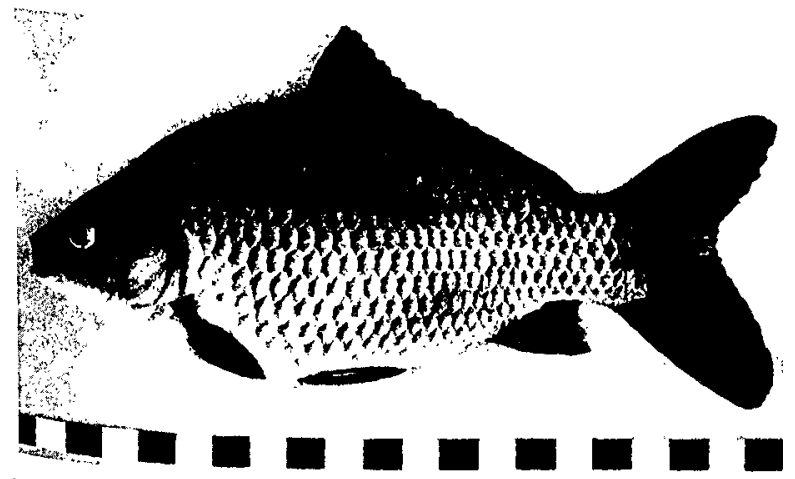

Fig. 7. In vitro erzeugter Karpfen-Goldfisch-Bastard fangreiche Züchtungsvorhaben zu. In unserem Institut sind die Züchtung von Karpfen auf Leistung und auf Grätenfreiheit vorrangige Ziele. Durch Bildung von Inzuchtlinien soll ein Hybridkarpfen von erhöhter Frohwüchsigkeit angestrebt werden. Mit Hilfe einer Röntgenfernsehapparatur können wir die Selektionsarbeit hinsichtlich der Anzahl der Zwischenmuskelgräten durchführen und haben dabei bereits erhebliche Variationen festgestellt [26]. Besonders nachdem uns in letzter Zeit mehrere Fälle von grätenfreien Karpfen gemeldet wurden, müßte die Arbeit zur Züchtung eines zwischenmuskelgrätenfreien Karpfens verstärkt werden. Allerdings sind hierfür beträchtliche Mittel notwendig.

\section{Anwendung}

Die Warmwasser-Fischzucht kann für zahlreiche Nutzfischarten Anwendung finden. Wir führen z.Z. auch Haltungs- und Fütterungsversuche mit Aalen, Stören, Welsen und auch Krebsen durch. Verschiedene Arten tropischer Speisefische könnten als neue Nutzfischarten in Kultur genommen werden. Die neuen Verfahren der Haltung, Fütterung und Züchtung bieten der Warmwasser-Fischzucht zahlreiche Anwendungsmöglichkeiten in Wissenschaft und Praxis. Autökologie, Ernährungsphysiologie, Fortpflanzungsbiologie und Züchtung können bei Nutzfischen erstmals unter standardisierten, kontrollierbaren und steuerbaren Verhältnissen untersucht werden. Das Versuchstier Fisch wird eine neue Bedeutung gewinnen, besonders, da mit genetisch einheitlichem Material gearbeitet werden kann. Zur Lösung der zukünftigen Welternährungsprobleme stellt die Warmwasser-Fischzucht einen an Bedeutung wachsenden Beitrag dar. Bei Anwendung des Wasserkreislaufsystems bietet sie die Möglichkeit, der steigenden Umweltverschmutzung auszuweichen. Die deutsche Teichwirtschaft, die unter klimatischen Nachteilen und Gewässervergiftungen leidet, hat bereits eine erste praktische Hilfe durch die WarmwasserFischzucht erhalten: In unserer Anlage im zeitigen Frühling erzeugte Karpfenbrut wurde 1970 in größerem Stil an teichwirtschaftliche Betriebe abgegeben und erbrachte bei der Herbstabfischung erhebliche Mehrerträge gegenüber den dort üblichen Produktionsmethoden [27]. Eine Neuorientierung der deutschen Teichwirtschaft [28] wird als Konsequenz der bisherigen Warmwasser-Fischzucht-Arbeiten diskutiert und soll den Leistungsstandard der Binnenfischerei verbessern [29].

Die zahlreichen Forschungsvorhaben der oben erwähnten Disziplinen können nur unter Mitwirkung mehrerer Wissenschaftler dieser Fachrichtung konsequent bearbeitet werden. Die bei uns entwickelten und im kleinen Rahmen angewandten Verfahren können wir bald unter besseren Bedingungen erproben und erweitern. Die Stiftung Volkswagenwerk hat den Bau ciner ,Arbeitsstätte zur Biologie der Fische" im Werte von 1 Mill. DM ermöglicht, die in Kürze in Betrieb genommen wird.

Die Photos fertigte dankenswerterweise Herr K. Engelhardt an, die Zeichnungen Frau A. Mollwo und Herr W. Szablewski.

[1] Thurow, F., in: Jahresber. Forschung im Geschäftsbereich des Bundesministers für Ernährung, Landwirtschaft und Forsten, Teil C, C 32 (1969). - [2] Schubert, K.: Informat. Fischwirtschaft 17, 195 (1970). - [3] Riedel, D.: Fischwirt 21, 10 (1971). - [4] v. Sengbusch, R.: Züchter 33, 284 
(1963). - [5] v. Sengbusch, R., in: Vortragsveranstaltung über neue Methoden der Fischzüchtung und -haltung. Hamburg: MPI f. Kulturpflanzenzüchtung 1967. - [6] Langhans, V. Nachrichtenbl. Fischzucht Fischerei 1, 122 (1928). [7] Krupauer, V.: Rybářství H. 6, 85 (1963). - [8] v. Sengbusch, R., Meske, Ch., Szablewski, W.: Experientia 21, 614 (1965). - [9] v. Sengbusch, R., et al.: Z. Fischerei 15 N. F., 45 (1967). - [10] Meske, Ch.: Fischwirt 16, 309 (1966) [11] Koßmann, H.: ibid. 20, 255 (1970). - [12] Schäperclaus, W.: Lehrbuch der Teichwirtschaft. Berlin-Hamburg: Paul Parey 1961 - [13] Jancarik, A.: Z. Fischerei 12 N. F., 602 (1964). - [14] Lühr, B,, in: Vortragsveranstaltung über neue Methoden der Fischzüchtung und -haltung. Hamburg: MPI f. Kulturpflanzenzüchtung 1967. - [15] Meske, Ch.: Fischwirt 18, 310 (1968). - [16] Meyer-Waarden, P. F., in: Der Aal. Hamburg: A. Keune 1965. - [17] Müller, H.: Dtsch.
Fischerei-Z, 14, 1 (1967). - [18] Meske, Ch.: Arch. Fischereiwiss. 20, 26 (1969). - [19] Suworow, E.: Grundlagen der Ichthyologie. Moskau 1948. - [20] Ihering, G.: Zool. Anz. 3 (1935). - [21] Gerbilski, H. L.: Vesti. Leningrad un.-ta Nr. (zitiert nach Woynárovich 1963). (1951). - [22] Woynárovich, E.. Beitr. Gewässerforsch. 4, 210 (1964). - [23] Meske, Ch., et al.: Theoret. Appl. Genetics 38, 47 (1968). [24] Koßmann, H. - Zuchthygiene, im Druck. - [25] Meske, Ch., Lühr, B., Szablewski, W.: Naturwissenschaften 54, 291 (1967). - [26] v. Sengbusch, R., Meske, Ch.: Züchter 37, 271 (1967). - [27] Koßmann, H., Szablewski, W.: Fischwirt 21, 49 (1971). - [28] Denkschrift der DLG-Tierzuchtabteilung (1967). - [29] Meske, Ch. Fischwirt 19, 244 (1969).

Eingegangen am 22. Februar 1971 